\title{
磺酸功能化纳米 $\gamma-\mathrm{Al}_{2} \mathrm{O}_{3}$ 催化下合成硫醚类化合物
}

\author{
李卫林*,a 李 爽 ${ }^{b}$ 武利强 ${ }^{a}$ 间福林 ${ }^{a}$ \\ $\left({ }^{a}\right.$ 新乡医学院药学院 新乡 453003) \\ ( ${ }^{b}$ 新乡医学院基础医学院 新乡 453003)
}

\begin{abstract}
摘要 在磺酸功能化纳米 $\gamma-\mathrm{Al}_{2} \mathrm{O}_{3}$ 催化下, 以硫醇或硫酚和醇类化合物为原料, 在室温、无溶剂条件下, 高收率地实现 了一系列硫醂类化合物的合成, 产物结构经 ${ }^{1} \mathrm{H} \mathrm{NMR},{ }^{13} \mathrm{C} N \mathrm{NR}$ 和元素分析进行了表征.

关键词 硫醚类化合物; 磺酸基功能化的纳米 $\gamma-\mathrm{Al}_{2} \mathrm{O}_{3}$; 合成
\end{abstract}

\section{Sulfonic Acid Functionalized Nano $\mathrm{Y}-\mathrm{Al}_{2} \mathrm{O}_{3}$ Catalyzed Synthesis of Thioethers}

\author{
Li, Weilin ${ }^{*, a} \quad$ Li, Shuang $^{b} \quad$ Wu, Liqiang ${ }^{a} \quad$ Yan, Fulin $^{a}$ \\ $\left({ }^{a}\right.$ School of Pharmacy, Xinxiang Medical University, Xinxiang 45300) \\ $\left({ }^{b}\right.$ School of Basic Medical Sciences, Xinxiang Medical Univirsity, Xinxiang 453003)
}

\begin{abstract}
A series of thioethers were synthesized efficiently in high yields by reaction of thiols with alcohols in the presence of sulfonic acid functionalized nano $\gamma-\mathrm{Al}_{2} \mathrm{O}_{3}$ at room temperature under solvent-free conditions. All products were characterized by ${ }^{1} \mathrm{H}$ NMR, ${ }^{13} \mathrm{C}$ NMR spectra and elemental analysis.

Keywords thioethers; sulfonic acid functionalized nano $\gamma-\mathrm{Al}_{2} \mathrm{O}_{3}$; synthesis
\end{abstract}

硫醚类化合物是有机合成化学中的一种重要的中 间体，在精化细工、农药和其它的相关行业中有着广泛 的应用 ${ }^{[1 \sim 5]}$, 同时硫醚也是某些生物活性物质和药物的 关键结构片断 ${ }^{[6-9]}$. 目前主要的合成方法有以下 5 种: (1)

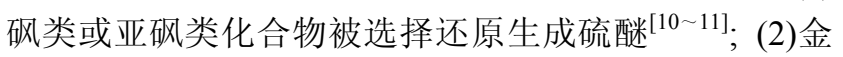
属参与 $\mathrm{C}-\mathrm{S}$ 键偶联 ${ }^{[12 \sim 15]}$; (3)硫醇硫酚对烯烃加成 ${ }^{[16 ~ 17] ; ~}$ (4)利用 Mitsunobu 反应构建 $\mathrm{C}-\mathrm{S}$ 键 ${ }^{[17]} ;(5)$ 硫亲核试剂 对醇/卤代烃的亲核取代反应 ${ }^{[18]}$.

为进一步研究硫醚衍生物的合成及其生物活性, 本 实验以磺酸功能化的纳米 $\gamma-\mathrm{Al}_{2} \mathrm{O}_{3}$ 为催化剂, 在室温和 无溶剂的条件下, 应用硫醇或硫酚对各种醇进行 $S$-烷基 化反应，高收率地实现了一系列硫醚类化合物的合成. 该方法不失为一种快捷高效硫醚类化合物的绿色合成 新方法. 目标化合物的结构通过 ${ }^{1} \mathrm{H} N M R,{ }^{13} \mathrm{C} N M R$ 和 元素分析进行了表征. 合成路线及结果见 Eq. 1 和表 1.

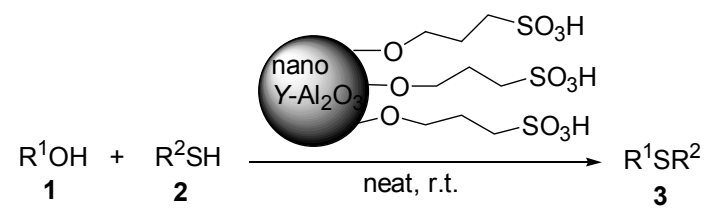

\section{1 结果与讨论}

\section{1 催化剂的合成与表征}

纳米材料作为催化剂, 具有多孔性、比表面较大、 孔隙率高、高分散度、热稳定性和表面酸性、催化效率 高的特点, 被广泛应用各种有机反应中 ${ }^{[19-22]}$. 磺酸功能 化基的纳米 $\gamma-\mathrm{Al}_{2} \mathrm{O}_{3}$ 可方便的由 Eq. 2 所示的方法制的. 其结构特点我们通过红外光谱、热重量分析、扫描电镜 分析与透镜电镜分析对其结构进行了表征.

红外光谱显示(图 1), 与纳米 $\gamma-\mathrm{Al}_{2} \mathrm{O}_{3}$ 的红外光谱相

\footnotetext{
*E-mail: linweilin1977@163.com

Received November 1, 2012; revised December 18, 2012; published online January 4, 2013.

Project supported by the Foundation of Henan Educational Committee (No. 12B350005).

河南省教育厅科学技术研究重点(No. 12B350005)资助项目.
} 
表 1 磺酸功能化的纳米 $\gamma-\mathrm{Al}_{2} \mathrm{O}_{3}$ 催化合成硫醚衍类化合物 ${ }^{a}$

Table 1 Synthesis of thioethers using sulfonic acid functionalized nano $\gamma-\mathrm{Al}_{2} \mathrm{O}_{3}$

\begin{tabular}{|c|c|c|c|c|c|}
\hline Entry & $\mathrm{R}^{1}$ & $\mathrm{R}^{2}$ & Product & Time/min & Yield $^{b} / \%$ \\
\hline 1 & $\mathrm{Bn}$ & $\mathrm{Ph}$ & $3 a$ & 13 & 90 \\
\hline 2 & $\mathrm{Bn}$ & 4- $\mathrm{CH}_{3} \mathrm{C}_{6} \mathrm{H}_{4}$ & $3 \mathbf{b}$ & 10 & 93 \\
\hline 3 & $\mathrm{Bn}$ & $4-\mathrm{CH}_{3} \mathrm{OC}_{6} \mathrm{H}_{4}$ & $3 c$ & 12 & 91 \\
\hline 4 & $\mathrm{Bn}$ & $4-\mathrm{ClC}_{6} \mathrm{H}_{4}$ & 3d & 25 & 68 \\
\hline 5 & $\mathrm{Bn}$ & $4-\mathrm{NO}_{2} \mathrm{C}_{6} \mathrm{H}_{4}$ & $3 e$ & 35 & 56 \\
\hline 6 & $\mathrm{Bn}$ & 2-Naphthyl & $3 f$ & 12 & 89 \\
\hline 7 & $\mathrm{Bn}$ & 2-Benzimidazoyl & $3 g$ & 16 & 82 \\
\hline 8 & $\mathrm{Bn}$ & 1-Decyl & $3 \mathbf{h}$ & 28 & 72 \\
\hline 9 & $\mathrm{Bn}$ & $\mathrm{Cy}$ & $3 \mathbf{i}$ & 24 & 81 \\
\hline 10 & 4- $\mathrm{CH}_{3} \mathrm{C}_{6} \mathrm{H}_{4} \mathrm{CH}_{2}$ & $\mathrm{Ph}$ & $3 \mathbf{j}$ & 10 & 89 \\
\hline 11 & $4-\mathrm{ClC}_{6} \mathrm{H}_{4} \mathrm{CH}_{2}$ & $\mathrm{Ph}$ & $3 \mathbf{k}$ & 26 & 78 \\
\hline 12 & $4-\mathrm{NO}_{2} \mathrm{C}_{6} \mathrm{H}_{4} \mathrm{CH}_{2}$ & $\mathrm{Ph}$ & 31 & 45 & 58 \\
\hline 13 & $\mathrm{Ph}_{2} \mathrm{CH}$ & $\mathrm{Ph}$ & $3 \mathrm{~m}$ & 18 & 95 \\
\hline 14 & 1-Adamantanyl & 4- $\mathrm{CH}_{3} \mathrm{C}_{6} \mathrm{H}_{4}$ & $3 n$ & 20 & 94 \\
\hline 15 & $\mathrm{PhCH}=\mathrm{CH}$ & $4-\mathrm{CH}_{3} \mathrm{C}_{6} \mathrm{H}_{4}$ & 30 & 15 & 90 \\
\hline 16 & $\mathrm{PhCH}=\mathrm{CH}$ & 4- $\mathrm{NO}_{2} \mathrm{C}_{6} \mathrm{H}_{4}$ & $3 p$ & 28 & 62 \\
\hline
\end{tabular}

质应条件: 硫醇/硫酚 $(1 \mathrm{mmol})$, 醇 $(1 \mathrm{mmol})$, 功能化磺酸基纳米 $\gamma-\mathrm{Al}_{2} \mathrm{O}_{3}(200 \mathrm{mg})$, 室温, 无溶剂. ${ }^{b}$ 分离后的产率.

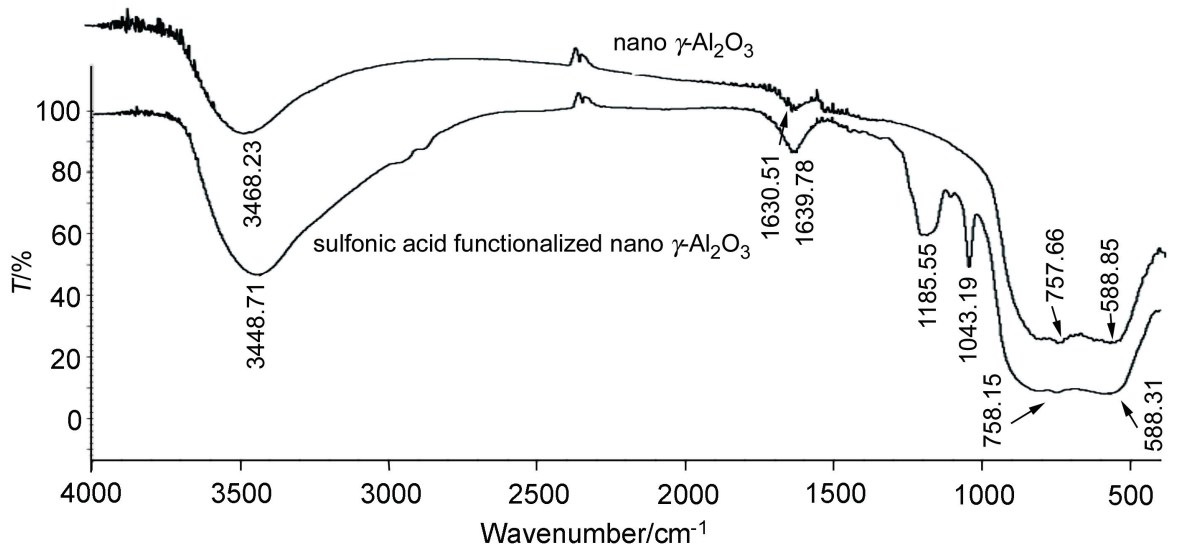

图 1 纳米 $\gamma-\mathrm{Al}_{2} \mathrm{O}_{3}$ 与磺酸功能化纳米 $\gamma-\mathrm{Al}_{2} \mathrm{O}_{3}$ 的红外光谱

Figure 1 FT-IR spectra of nano $\gamma-\mathrm{Al}_{2} \mathrm{O}_{3}$ and sulfonic acid functionalized nano $\gamma-\mathrm{Al}_{2} \mathrm{O}_{3}$

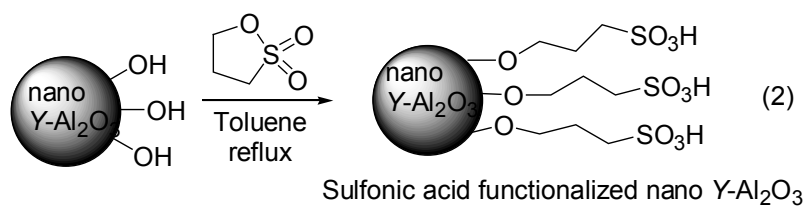

比较, 磺酸功能化纳米 $\gamma-\mathrm{Al}_{2} \mathrm{O}_{3}$ 在红外图谱中 $589 \mathrm{~cm}^{-1}$ 和 $758 \mathrm{~cm}^{-1}$ 明显多了二个特征吸收峰, 这主要是 $\gamma-\mathrm{Al}_{2} \mathrm{O}_{3}$ 的 $\mathrm{Al}-\mathrm{O}$ 键伸缩振动引起吸收, 而且在 1043 和 $1187 \mathrm{~cm}^{-1}$ 出现了 $\mathrm{S}-\mathrm{O}$ 键的伸缩振动吸收, 在 3444 $\mathrm{cm}^{-1}$ 处出现的红外吸收, 归属于 $\mathrm{SO}_{3} \mathrm{H}$ 中的 $\mathrm{OH}$ 红外吸 收峰. 从结果可知, 纳米 $\gamma-\mathrm{Al}_{2} \mathrm{O}_{3}$ 与 1,3 -丙磺内酯反应使 丙磺酸基成功的键合在纳米 $\gamma-\mathrm{Al}_{2} \mathrm{O}_{3}$ 上.

利用热重量分析对纳米 $\gamma-\mathrm{Al}_{2} \mathrm{O}_{3}$ 和磺酸功能化纳米 $\gamma-\mathrm{Al}_{2} \mathrm{O}_{3}$ 的稳定性也进行了考察, 从图 2 可以看出, 在 $150{ }^{\circ} \mathrm{C}$ 由于纳米 $\gamma-\mathrm{Al}_{2} \mathrm{O}_{3}$ 和磺酸功能化纳米 $\gamma-\mathrm{Al}_{2} \mathrm{O}_{3}$ 的表
面水分失去, 在热重量曲线上都出现明显的下降, 当温 度 230 960 ${ }^{\circ} \mathrm{C}$ 由于磺酸功能化纳米 $\gamma-\mathrm{Al}_{2} \mathrm{O}_{3}$ 表面所有 共价键的断裂, 使重量下降的比较明显. 从温度小于 $328{ }^{\circ} \mathrm{C}$ 的这个肩峰观察发现, 其重量的下降是磺酸功能 化纳米 $\gamma-\mathrm{Al}_{2} \mathrm{O}_{3}$ 表面烷基化磺酸分解所引起. 根据热重 量分析曲线可知, 在磺酸功能化纳米 $\gamma-\mathrm{Al}_{2} \mathrm{O}_{3}$ 表面, 磺酸 基的数量为 $0.78 \mathrm{mmol} \cdot \mathrm{g}^{-1}$, 这一点与离子交换 $\mathrm{pH}$ 值测 定结果相一致.

利用扫描电镜对纳米 $\gamma-\mathrm{Al}_{2} \mathrm{O}_{3}$ 和磺酸功能化纳米 $\gamma-\mathrm{Al}_{2} \mathrm{O}_{3}$ 的形态学及粉末大小进行的考察可以看出(图 3), 纳米 $\gamma-\mathrm{Al}_{2} \mathrm{O}_{3}$ 和磺酸功能化的纳米 $\gamma-\mathrm{Al}_{2} \mathrm{O}_{3}$ 颗粒呈球形或 半球形, 粒度分布比较均匀, 这与 $\gamma-\mathrm{Al}_{2} \mathrm{O}_{3}$ 纳米微粒晶体 特性相一致. 图 3a $3 b$ 中出现的较大晶粒是由于纳米 晶粒之间团聚形成的. 进一步利用透射电镱对其进行研 


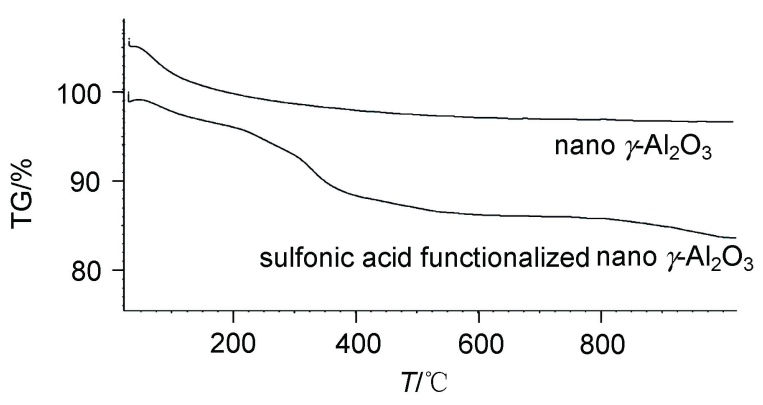

图 2 纳米 $\gamma-\mathrm{Al}_{2} \mathrm{O}_{3}$ 与磺酸功能化纳米 $\gamma-\mathrm{Al}_{2} \mathrm{O}_{3}$ 的热重量分析 Figure 2 TG analyses of nano $\gamma-\mathrm{Al}_{2} \mathrm{O}_{3}$ (up) and nano $n$-propylsulfonated $\gamma-\mathrm{Al}_{2} \mathrm{O}_{3}$

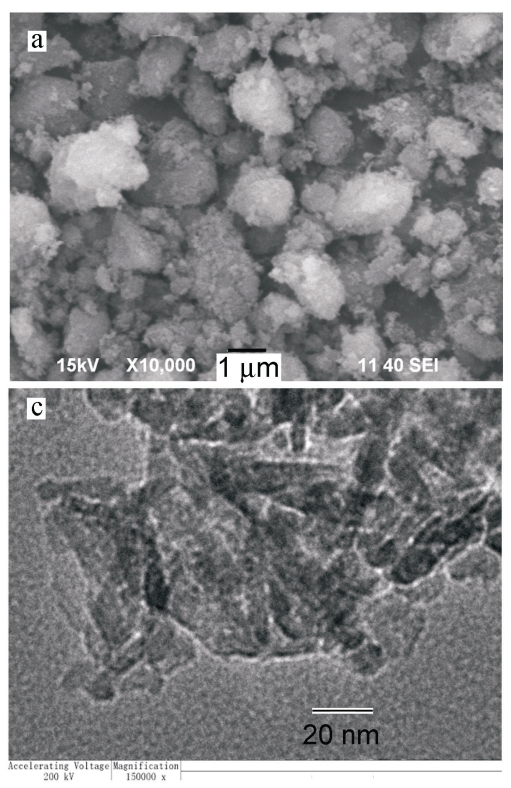

究, 图 3c $\sim 3 \mathrm{~d}$ 结果显示纳米 $\gamma-\mathrm{Al}_{2} \mathrm{O}_{3}$ 和磺酸功能化纳米 $\gamma-\mathrm{Al}_{2} \mathrm{O}_{3}$ 微粒的大小在 $10 \sim 20 \mathrm{~nm}$ 之间.

\section{2 催化剂用量的优化}

为了优化反应条件, 我们以芐醇与苯硫酚反应生成 芐基苯基硫醚为例, 考察了各种催化剂和不同磺酸功能 化纳米 $\gamma-\mathrm{Al}_{2} \mathrm{O}_{3}$ 用量对其产率的影响(表 2).

从表 2 可以看出功能化磺酸基纳米 $\gamma-\mathrm{Al}_{2} \mathrm{O}_{3}$ 作催化 剂为 $200 \mathrm{mg} / \mathrm{mmol}$, 芐基苯基硫醚的产率最高, 再增加 催化剂的用量对苄基苯基硫醚的产率影响不是很大，使 用其它催化剂，从反应时间和产率来说，效果没有功能 化磺酸基纳米 $\gamma-\mathrm{Al}_{2} \mathrm{O}_{3}$ 好.

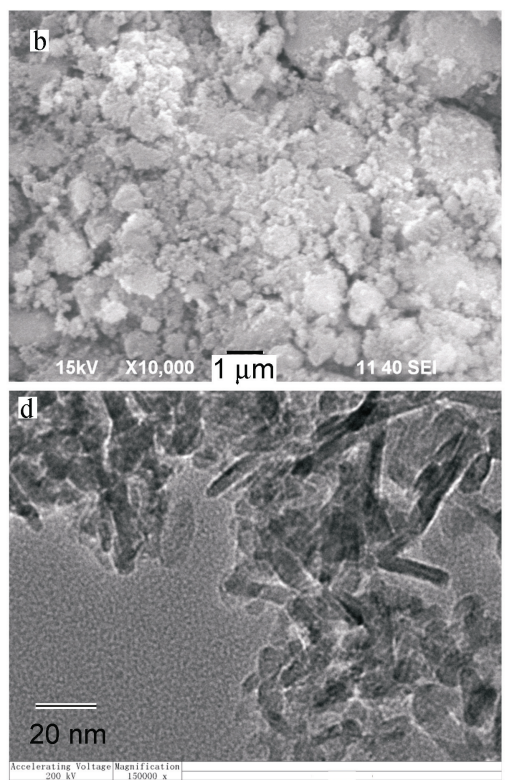

图 3 纳米 $\gamma-\mathrm{Al}_{2} \mathrm{O}_{3}$ (a) 和磺酸功能化纳米 $\gamma-\mathrm{Al}_{2} \mathrm{O}_{3}$ (b) 的扫描电镜分析, 纳米 $\gamma-\mathrm{Al}_{2} \mathrm{O}_{3}$ (c) 和磺酸功能化纳米 $\gamma-\mathrm{Al}_{2} \mathrm{O}_{3}(\mathrm{~d})$ 的透视电镜分 析

Figure 3 SEM images of nano $\gamma-\mathrm{Al}_{2} \mathrm{O}_{3}$ (a) and sulfonic acid functionalized nano $\gamma-\mathrm{Al}_{2} \mathrm{O}_{3}$ (b), TEM images of nano $\gamma-\mathrm{Al}_{2} \mathrm{O}_{3}$ (c) and sulfonic acid functionalized nano $\gamma-\mathrm{Al}_{2} \mathrm{O}_{3}$ (d)

表 2 催化剂种类和用量对 3a 产率的影响 ${ }^{a}$

Table 2 Catalyst optimization for the synthesis 3a

\begin{tabular}{cllcc}
\hline \multicolumn{1}{c}{ Catalyst } & Mass $/ \mathrm{mg}$ & Time/min & Yield/\% \\
\hline 1 & Sulfonic acid functionalized nano $\gamma-\mathrm{Al}_{2} \mathrm{O}_{3}$ & 0 & 120 & 0 \\
2 & Sulfonic acid functionalized nano $\gamma-\mathrm{Al}_{2} \mathrm{O}_{3}$ & 25 & 20 & 48 \\
3 & Sulfonic acid functionalized nano $\gamma-\mathrm{Al}_{2} \mathrm{O}_{3}$ & 50 & 15 & 56 \\
4 & Sulfonic acid functionalized nano $\gamma-\mathrm{Al}_{2} \mathrm{O}_{3}$ & 100 & 15 & 84 \\
5 & Sulfonic acid functionalized nano $\gamma-\mathrm{Al}_{2} \mathrm{O}_{3}$ & 200 & 10 & 92 \\
6 & Sulfonic acid functionalized nano $\gamma-\mathrm{Al}_{2} \mathrm{O}_{3}$ & 300 & 10 & 86 \\
7 & Sulfonic acid functionalized nano $\gamma-\mathrm{Al}_{2} \mathrm{O}_{3}$ & 350 & 10 & 89 \\
8 & $\mathrm{CH}_{3} \mathrm{SO}_{3} \mathrm{H}$ & 200 & 45 & 62 \\
9 & $\mathrm{Alumina}_{2}$ & 200 & 60 & 52 \\
10 & $\mathrm{Nano} \gamma-\mathrm{Al}_{2} \mathrm{O}_{3}$ & 200 & 120 & 12 \\
11 & $p-\mathrm{TsOH}_{2}$ & 200 & 30 & 59 \\
12 & $\mathrm{H}_{2} \mathrm{SO}_{4}$ & 200 & 60 & 24 \\
\hline
\end{tabular}

${ }^{a}$ 反应条件: 苯硫酚 $(1 \mathrm{mmol})$, 芐醇 $(1 \mathrm{mmol})$, 室温, 无溶剂. 


\section{3 反应普遍性研究}

在优化的反应条件的基础上, 我们以磺酸功能化纳 米 $\gamma-\mathrm{Al}_{2} \mathrm{O}_{3}$ 为催化剂, 在室温和无溶剂的条件下, 高收率 的合成了一系列的硫醚衍生物, 产物结构经 ${ }^{1} \mathrm{H}$ NMR,

${ }^{13} \mathrm{C} \mathrm{NMR}$, 元素分析等进行了表征. 此外, 我们也注意 到无论是芳香硫酚还是环状、直链硫醇都可以和醇类化 合物很好的反应生成目标化合物(表 1).

\section{4 催化剂的可重复性}

以苠醇与苯硫酚反应生成苠基苯基硫醚为例, 考察 了催化剂回收后再使用对其产率的影响. 在每次反应 后，通过简单的过滤，乙醇洗涤，100 ${ }^{\circ} \mathrm{C}$ 干燥 $30 \mathrm{~min}$ 后， 催化剂可被用于下次实验. 在相同的实验条件下, 催化 剂反复使用 3 次后, 催化剂还保持较高的活性, 芐基苯基 硫醚的收率分别是 $92 \%, 89 \%, 86 \%$.

\section{2 结论}

在室温、无溶剂条件下, 以磺酸功能化纳米 $\gamma-\mathrm{Al}_{2} \mathrm{O}_{3}$ 作为催化剂, 硫酚/硫醇和醇类化合物反应, 高收率地实 现了一系列硫醚类衍生物的合成, 这个方法具有简单高 效, 后处理方便的优点, 它为合成硫醚衍生物类化合物 提供了一条新的途径.

\section{3 实验部分}

\section{1 仪器与试剂}

化合物熔点采用毛细管法测定, 温度计未校正; ${ }^{1} \mathrm{H}$ NMR 由 Bruker AV-400 核磁共振仪测定(TMS 为内标, $\mathrm{CDCl}_{3}$ 为溶剂); 红外光谱(FT-IR)由 Nicolet 6700 仪器测 定; 元素分析采用 Vario EL III 元素分析仪测定; 扫描电 镜分析在 JSM-6390LV 型仪器上完成. 透射电镜分析在 JEM 2100 仪器 $(150 \mathrm{kV})$ 上测定; 热重量分析曲线(TGA) 在 DT-40 仪器上完成. 纳米 $\gamma-\mathrm{Al}_{2} \mathrm{O}_{3}$ 购自阿拉丁试剂公 司, 所用其它试剂为市售化学纯或分析纯.

\section{2 磺酸功能化的纳米 $\gamma-\mathrm{Al}_{2} \mathrm{O}_{3}$ 催化剂的合成}

将 $6 \mathrm{~g}$ 纳米 $\gamma-\mathrm{Al}_{2} \mathrm{O}_{3}$ 粉末加至 $1000 \mathrm{~mL}$ 圆底烧瓶中,

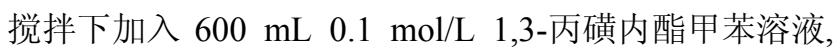
回流反应 $48 \mathrm{~h}$ 后, 放置冷却, 离心分离得磺酸功能化的 纳米 $\gamma-\mathrm{Al}_{2} \mathrm{O}_{3}$.

\section{3 离子交换 $\mathrm{pH}$ 值测定}

配置 $25 \mathrm{~mL}$ 的 $1 \mathrm{~mol} / \mathrm{L} \mathrm{NaCl}$ 水溶液, 测定其 $\mathrm{pH}$ 为 5.93 , 将 $500 \mathrm{mg}$ 磺化的 $\gamma-\mathrm{Al}_{2} \mathrm{O}_{3}$ 催化剂缓慢加至 $\mathrm{NaCl}$ 水溶液, 搅拌 $2 \mathrm{~h}$ 后, 测定其 $\mathrm{pH}$ 值为 1.81 , 这相当于每 克纳米 $\gamma-\mathrm{Al}_{2} \mathrm{O}_{3}$ 含 $0.78 \mathrm{mmol} \mathrm{SO}_{3} \mathrm{H}$.

\section{4 硫醚衍生物类化合物的合成}

将 $1 \mathrm{mmol}$ 硫醇或硫酚和 $1 \mathrm{mmol}$ 醇加到 $250 \mathrm{~mL}$ 三 口瓶中, 搅拌下加入 $200 \mathrm{mg}$ 磺酸功能化的纳米 $\gamma-\mathrm{Al}_{2} \mathrm{O}_{3}$, 在室温下搅拌反应 10 45 min 后, 产物加 $25 \mathrm{~mL}$ 水和 $100 \mathrm{~mL}$ 乙酸乙酯, 过滤, 有机层用无水 $\mathrm{Na}_{2} \mathrm{SO}_{4}$ 干燥, 过滤，蒸出溶剂，硅胶柱分离纯化，得化合物 3.

苠基苯基硫醚(3a): 白色结晶，m.p. $46 \sim 47{ }^{\circ} \mathrm{C} ;{ }^{1} \mathrm{H}$ NMR $\left(\mathrm{CDCl}_{3}, 400 \mathrm{MHz}\right) \delta: 3.49(\mathrm{~s}, 2 \mathrm{H}), 7.12 \sim 7.23(\mathrm{~m}$, $10 \mathrm{H}) ;{ }^{13} \mathrm{C} \mathrm{NMR}\left(\mathrm{CDCl}_{3}, 100 \mathrm{MHz}\right) \delta: 137.7,136.9,129.8$, $129.4,128.8,127.7,127.5,126.6,43.5$. Anal. calcd for $\mathrm{C}_{13} \mathrm{H}_{12} \mathrm{~S}$ : C 77.95, H 6.04, H 16.01; found C 77.92, H 6.06, H 16.04 .

苠基-4-甲基苯基硫醚(3b)：白色结晶，m.p. 44.1 $45.0{ }^{\circ} \mathrm{C} ;{ }^{1} \mathrm{H}$ NMR $\left(\mathrm{CDCl}_{3}, 400 \mathrm{MHz}\right) \delta: 2.21(\mathrm{~s}, 3 \mathrm{H}), 4.13$ (s, 2H), $7.23(\mathrm{~d}, J=8.0 \mathrm{~Hz}, 2 \mathrm{H}), 7.05$ (d, $J=8.0 \mathrm{~Hz}, 2 \mathrm{H})$, $7.25 \sim 7.63(\mathrm{~m}, 5 \mathrm{H}) ;{ }^{13} \mathrm{C}$ NMR $\left(100 \mathrm{MHz}, \mathrm{CDCl}_{3}\right) \delta$ : $136.7,135.4,131.4,129.6,128.5,127.9,127.4,126.0$, 125.9, 38.7, 20.0. Anal. calcd for $\mathrm{C}_{14} \mathrm{H}_{14} \mathrm{~S}: \mathrm{C} 78.46, \mathrm{H}$ 6.58, S 14.96; found C 78.45, H 6.60, S 14.94.

苠基-4-甲氧基苯基硫醚 (3c): 白色结晶, m.p. $47.2 \sim 49.0{ }^{\circ} \mathrm{C} ;{ }^{1} \mathrm{H}$ NMR $\left(\mathrm{CDCl}_{3}, 400 \mathrm{MHz}\right) \delta: 3.88(\mathrm{~s}$, 2H), 3. 62 (s, 3H), $7.33(\mathrm{~d}, J=8.8 \mathrm{~Hz}, 2 \mathrm{H}), 6.83(\mathrm{~d}, J=8.8$ $\mathrm{Hz}, 2 \mathrm{H}), 7.28 \sim 7.35(\mathrm{~m}, 3 \mathrm{H}), 7.30 \sim 7.35(\mathrm{~m}, 2 \mathrm{H}), 6.64 \sim$ $6.70(\mathrm{~m}, 2 \mathrm{H}) ;{ }^{13} \mathrm{C} \mathrm{NMR}\left(100 \mathrm{MHz}, \mathrm{CDCl}_{3}\right) \delta: 158.1$, 137.0, 132.9, 127.8, 127.3, 125.9, 125.0, 113.3, 54.2, 40.1. Anal. calcd for $\mathrm{C}_{14} \mathrm{H}_{14} \mathrm{OS}$ : C 73.01, H 6.13, S 13.92; found C 73.05, H 6.10, S 13.90.

苠基-4-氯苯基硫醚(3d)：白色结晶, m.p. 95.2 96.0 ${ }^{\circ} \mathrm{C} ;{ }^{1} \mathrm{H}$ NMR $\left(\mathrm{CDCl}_{3}, 400 \mathrm{MHz}\right) \delta: 4.07$ (s, 2H), 7.43 (d, $J=8.1 \mathrm{~Hz}, 2 \mathrm{H}), 7.23(\mathrm{~d}, J=8.2 \mathrm{~Hz}, 2 \mathrm{H}), 7.31 \sim 7.25(\mathrm{~m}$, $3 \mathrm{H}), 7.30 \sim 7.33(\mathrm{~m}, 2 \mathrm{H}), 7.20(\mathrm{~s}, 4 \mathrm{H}) ;{ }^{13} \mathrm{C}$ NMR $(100$ $\left.\mathrm{MHz}, \mathrm{CDCl}_{3}\right) \delta: 137.1,134.7,132.5,131.5,129.0,128.8$, 128.6, 127.3, 39.3. Anal. calcd for $\mathrm{C}_{13} \mathrm{H}_{11} \mathrm{ClS}$ : $\mathrm{C} 66.51, \mathrm{H}$ 4.72, S 13.66; found C66.53, H 4.74, S 13.63.

苠基-4-硝基苯基硫梄(3e)：白色结晶，m.p. 128.1 $129.0{ }^{\circ} \mathrm{C} ;{ }^{1} \mathrm{H}$ NMR $\left(\mathrm{CDCl}_{3}, 400 \mathrm{MHz}\right) \delta: 4.16$ (s, 2H), 8.18 $(\mathrm{d}, J=8.0 \mathrm{~Hz}, 2 \mathrm{H}), 7.54(\mathrm{~d}, J=8.0 \mathrm{~Hz}, 2 \mathrm{H}), 7.38$ (d, $J=$ $7.9 \mathrm{~Hz}, 2 \mathrm{H}), 7.25 \sim 7.33(\mathrm{~m}, 3 \mathrm{H}) ;{ }^{13} \mathrm{C} \mathrm{NMR}(100 \mathrm{MHz}$, $\left.\mathrm{CDCl}_{3}\right) \delta: 147.2,145.1,135.4,129.7,128.8,128.7,127.8$, 126.5, 123.9, 36.9. Anal. calcd for $\mathrm{C}_{13} \mathrm{H}_{11} \mathrm{NO}_{2} \mathrm{~S}$ : C 63.65, H 4.52, N 5.71, S 13.07; found C 63.68, H 4.54, N 5.76, S 13.09 .

苠基-2-菜基硫醚(3f)：白色结晶，m.p. 89.2～90.0 ${ }^{\circ} \mathrm{C}$; ${ }^{1} \mathrm{H}$ NMR $\left(\mathrm{CDCl}_{3}, 400 \mathrm{MHz}\right) \delta: 7.74(\mathrm{~d}, J=8.0 \mathrm{~Hz}$, $1 \mathrm{H}), 7.70 \sim 7.66(\mathrm{~m}, 3 \mathrm{H}), 7.43 \sim 7.37(\mathrm{~m}, 3 \mathrm{H}), 7.31 \sim 7.19$ 
(m, 5H), 4.19 (s, 2H); ${ }^{13} \mathrm{C}$ NMR (100 MHz, $\left.\mathrm{CDCl}_{3}\right) \delta$ : 137.3, 133.9, 133.6, 131.8, 128.8, 128.5, 128.3, 127.6(2), 127.5, 127.2, 127.1, 126.4, 125.7, 38.8. Anal. calcd for $\mathrm{C}_{17} \mathrm{H}_{14} \mathrm{~S}$ : C 81.56, H 5.64, S 12.81; found C 81.58, H 4.65, $\mathrm{S} 12.85$.

芐基-2-苯并咪唑基硫醚(3g)：白色结晶, m.p. 175 $176{ }^{\circ} \mathrm{C} ;{ }^{1} \mathrm{H}$ NMR $\left(\mathrm{CDCl}_{3}, 400 \mathrm{MHz}\right) \delta: 4.54(\mathrm{~s}, 2 \mathrm{H}), 4.97$ (s, $1 \mathrm{H}), 7.12 \sim 7.08(\mathrm{~m}, 2 \mathrm{H}), 7.32 \sim 7.18(\mathrm{~m}, 3 \mathrm{H}), 7.40 \sim$ 7.49 (m, 4H); ${ }^{13} \mathrm{C}$ NMR $\left(\mathrm{CDCl}_{3}, 100 \mathrm{MHz}\right) \delta: 34.4,149.6$, 126.7, 128.6, 127.1, 137.5, 115.3, 123.5, 138.5, 152.4. Anal. calcd for $\mathrm{C}_{14} \mathrm{H}_{12} \mathrm{~N}_{2} \mathrm{~S}$ : C 69.97, H 5.03, N 11.66, S 13.34; found C 69.98, H 5.05, N 11. 67, H 13.36.

芐基癸基硫醚(3h): 无色油状物, ${ }^{1} \mathrm{H}$ NMR (400 $\left.\mathrm{MHz}, \mathrm{CDCl}_{3}\right) \delta: 7.16 \sim 7.21(\mathrm{~m}, 3 \mathrm{H}), 7.25 \sim 7.32(\mathrm{~m}, 2 \mathrm{H})$, $3.70(\mathrm{~s}, 2 \mathrm{H}), 2.40(\mathrm{t}, J=7.5 \mathrm{~Hz}, 2 \mathrm{H}), 1.57 \sim 1.51(\mathrm{~m}, 2 \mathrm{H})$, $1.32 \sim 1.25(\mathrm{~m}, 14 \mathrm{H}), 0.88(\mathrm{t}, J=6.7 \mathrm{~Hz}, 3 \mathrm{H}) ;{ }^{13} \mathrm{C} \mathrm{NMR}$ $\left(100 \mathrm{MHz}, \mathrm{CDCl}_{3}\right) \delta: 138.7,128.8,128.4,126.8,36.3$, 31.9, 31.4, 29.5(2), 29.3, 29.2, 28.9, 22.7, 14.1. Anal. calcd for $\mathrm{C}_{17} \mathrm{H}_{28} \mathrm{~S}$ : C 77. 20, H 10.67, S 12.12; found C 77.23, H 10.64, S 12.10 .

芐基环已基硫醚(3i): 无色油状物, ${ }^{1} \mathrm{H} \mathrm{NMR}\left(\mathrm{CDCl}_{3}\right.$, $400 \mathrm{MHz}) \delta: 0.79 \sim 0.84(\mathrm{~m}, 6 \mathrm{H}), 1.30 \sim 1.37(\mathrm{~m}, 4 \mathrm{H})$, $1.53 \sim 1.59(\mathrm{~m}, 1 \mathrm{H}), 4.19(\mathrm{~s}, 2 \mathrm{H}), 7.18 \sim 7.22(\mathrm{~m}, 3 \mathrm{H})$, $7.26 \sim 7.31(\mathrm{~m}, 2 \mathrm{H}),{ }^{13} \mathrm{C} \mathrm{NMR}\left(\mathrm{CDCl}_{3}, 100 \mathrm{MHz}\right) \delta: 24.9$, 27.6, 33.7, 36.5, 38.9, 127.9, 128.8, 129.1,139.4. Anal. calcd for $\mathrm{C}_{13} \mathrm{H}_{18} \mathrm{~S}$ : C 75.67, H 8.79, S 15.54; found $\mathrm{C}$ 75.63, H 8.76, S 15.52.

4-甲基苠基苯硫醚 $(\mathbf{3 j}$ ): 白色结晶, m.p. $68.2 \sim 69.0$ ${ }^{\circ} \mathrm{C} ;{ }^{1} \mathrm{H}$ NMR $\left(\mathrm{CDCl}_{3}, 400 \mathrm{MHz}\right) \delta$ : 2.49 (s, 3H), 4.09 (s, 2H) $7.12(\mathrm{~d}, J=8.3 \mathrm{~Hz}, 2 \mathrm{H}), 7.08$ (d, $J=8.3 \mathrm{~Hz}, 2 \mathrm{H}), 7.36$ (d, $J=8.0 \mathrm{~Hz}, 2 \mathrm{H}), 7.33 \sim 7.29(\mathrm{~m}, 1 \mathrm{H}$ ), $7.14 \sim 7.10(\mathrm{~m}$, $2 \mathrm{H}) ;{ }^{13} \mathrm{C}$ NMR $\left(\mathrm{CDCl}_{3}, 100 \mathrm{MHz}\right) \delta: 130.0,129.8,128.9$, 126.3, 114.0, 55.3, 38.5. Anal. calcd for $\mathrm{C}_{14} \mathrm{H}_{14} \mathrm{~S}$ : C 78.46, H 6.58, S 14.96; found C 78.42, H 6.59, S 14.98 .

4-氯芐基苯基硫醚(3k): 白色结晶, m.p. 79 $80{ }^{\circ} \mathrm{C}$; ${ }^{1} \mathrm{H}$ NMR $\left(\mathrm{CDCl}_{3}, 400 \mathrm{MHz}\right) \delta: 4.05$ (s, 2H), 7.39 (d, $J=$ $8.5 \mathrm{~Hz}, 2 \mathrm{H}), 7.28$ (d, $J=8.5 \mathrm{~Hz}, 2 \mathrm{H}), 7.39(\mathrm{~d}, J=8.2 \mathrm{~Hz}$, $2 \mathrm{H}), 7.31 \sim 7.33(\mathrm{~m}, 1 \mathrm{H}),, 7.20 \sim 7.18(\mathrm{~m}, 2 \mathrm{H},) ;{ }^{13} \mathrm{C} \mathrm{NMR}$ $\left(\mathrm{CDCl}_{3}, 100 \mathrm{MHz}\right) \delta: 136.08,132.87,130.62,130.20$, $130.06,128.87,128.59,126.63,38.48$. Anal. calcd for $\mathrm{C}_{13} \mathrm{H}_{11} \mathrm{ClS}$ : C 66.51, H 4.72, H 13.66; found C 63.54, H 4.75, H 13.68 .

4-硝基苄基苯基硫醚(31): 白色结晶, m.p. 72.2 $74.1{ }^{\circ} \mathrm{C} ;{ }^{1} \mathrm{H} \mathrm{NMR}\left(\mathrm{CDCl}_{3}, 400 \mathrm{MHz}\right) \delta$ : 4.11 (s, 2H), 8.12 (d, $J=8.9 \mathrm{~Hz}, 2 \mathrm{H}), 8.08$ (d, $J=8.8 \mathrm{~Hz}, 2 \mathrm{H}), 7.41$ (d, $J=$
$8.1 \mathrm{~Hz}, 2 \mathrm{H}), 7.34 \sim 7.37(\mathrm{~m}, 1 \mathrm{H}),, 7.21 \sim 7.24(\mathrm{~m}, 2 \mathrm{H}$, ${ }^{13} \mathrm{C} \mathrm{NMR}\left(\mathrm{CDCl}_{3}, 100 \mathrm{~Hz}\right) \delta: 145.6,136.6,132.5,131.1$, 129.6, 128.9, 127.4, 123.8, 39.1. Anal. calcd for $\mathrm{C}_{13} \mathrm{H}_{11} \mathrm{NO}_{2} \mathrm{~S}$ : C 63.65, H 4.52, N 5.71, S 13.07; found $\mathrm{C}$ 63.64, H 4.55, N 5.73, S 13.09.

二苯甲基苯基硫醚 $(\mathbf{3 m})$ : 白色结晶, m.p. 82 83.0 ${ }^{\circ} \mathrm{C} ;{ }^{1} \mathrm{H}$ NMR $\left(\mathrm{CDCl}_{3}, 400 \mathrm{MHz}\right) \delta: 5.46(\mathrm{~s}, 1 \mathrm{H}), 7.10 \sim$ $7.46(\mathrm{~m}, 15 \mathrm{H}) ;{ }^{13} \mathrm{C} \mathrm{NMR}\left(\mathrm{CDCl}_{3}, 100 \mathrm{MHz}\right) \delta: 52.2,127$, 127.6, 129.4, 130.1, 131.1, 133.3, 135.4, 145.3. Anal. calcd for $\mathrm{C}_{19} \mathrm{H}_{16} \mathrm{~S}$ : C 82.56, H 5.83, S 11.60; found C 82.57, H 5.87, S 11.65 .

(3S,5S,7S)-金刚烷-1-基-4 甲基苯基硫醚(3n): 无色 油状物; ${ }^{1} \mathrm{H} \mathrm{NMR}\left(\mathrm{CDCl}_{3}, 400 \mathrm{MHz}\right) \delta: 1.48 \sim 2.02(\mathrm{~m}$, $15 \mathrm{H}), 2.37$ (s, 3H), 7.05 (d, $J=8 \mathrm{~Hz}, 2 \mathrm{H}), 7.36$ (d, $J=8$ $\mathrm{Hz}, 2 \mathrm{H}) ;{ }^{13} \mathrm{C} \mathrm{NMR}\left(\mathrm{CDCl}_{3}, 100 \mathrm{MHz}\right) \delta: 21.0,26.2,28.3$, 37.4, 37.9, 128.5, 129.78, 129.88, 132.85, 137.7. Anal. calcd for $\mathrm{C}_{17} \mathrm{H}_{22} \mathrm{~S}$ : C 79.01, H 8. 58, S 12.41; found C 79.05, H 8. 56, S 12. 44.

4-甲基苯肉桂硫醚(3o): 无色油状物; ${ }^{1} \mathrm{H}$ NMR $\left(\mathrm{CDCl}_{3}, 400 \mathrm{MHz}\right) \delta: 2.31$ (s, 3H), $3.84(\mathrm{dd}, J=7.1,1.1$ $\mathrm{Hz}, 2 \mathrm{H}), 7.13 \sim 7.42(\mathrm{~m}, 9 \mathrm{H}), 6.51(\mathrm{~d}, J=15.9 \mathrm{~Hz}, 1 \mathrm{H})$, $6.32(\mathrm{~d}, J=15.9 \mathrm{~Hz}, 1 \mathrm{H}) ;{ }^{13} \mathrm{C} \mathrm{NMR}\left(\mathrm{CDCl}_{3}, 100 \mathrm{MHz}\right) \delta$ : $158.8,136.0,135.3,132.5,129.1,128.6,126.9,126.3$, 123.9, 123.0, 33.9, 21.3. Anal. calcd for $\mathrm{C}_{16} \mathrm{H}_{16} \mathrm{~S}: \mathrm{C} 79.95$, H 6.71, S 13.34; found C79. 97, H 6.73, S 13.36.

4-硝基苯肉桂硫醚(3p): 无色油状物; ${ }^{1} \mathrm{H}$ NMR $\left(\mathrm{CDCl}_{3}, 400 \mathrm{MHz}\right) \delta: 3.84(\mathrm{dd}, J=7.1,1.1 \mathrm{~Hz}, 2 \mathrm{H})$, $8.13 \sim 7.24$ (m, 9H), $6.53(\mathrm{~d}, J=15.9 \mathrm{~Hz}, 1 \mathrm{H}), 6.32(\mathrm{~d}, J=$ $15.9 \mathrm{~Hz}, 1 \mathrm{H}) ;{ }^{13} \mathrm{C}$ NMR $\left(100 \mathrm{MHz}, \mathrm{CDCl}_{3}\right) \delta: 152.8$, $134.0,132.3,131.5,129.1,128.6,126.9,126.3,123.9$, 123.0, 33.9; MS (ESI) $m / z: 271[\mathrm{M}]^{+}$. Anal. calcd for $\mathrm{C}_{15} \mathrm{H}_{13} \mathrm{NO}_{2} \mathrm{~S}$ : C 66.40, H 4.83, N 5.16, S11.82; found $\mathrm{C}$ 66.43, H 4.85, N, 5.18, S 11.85 .

\section{References}

[1] Moberg, H. I.; Omnaas, J. R. J. Am. Chem. Soc. 1985, 107, 2986.

[2] Hurby, V. J.; Al-Obeidi, F.; Kazmierski, W. Biochem. J. 1990, 268, 249.

[3] Kataoka, T.; Beusen, D. D.; Clark, J. D.; Yodo, M.; Marshall, G. R. Biopolymers 1992, 32, 1519.

[4] Hurby, V. J.; Bonner, G. G. Methods Mol. Biol. 1994, 35, 201.

[5] Li, Y.-Y.; Huang, Z.-G.; Xu, P.-F.; Zhang, Y.-N.; Wang, J.-B. Acta Chim. Sinica 2012, 70, 2024 (in Chinese).

(李玉叶, 黄重行, 许鹏飞, 张艳, 王剑波, 化学学报, 2012, 70, 2024.)

[6] Solladie, G. Synthesis 1981, 185.

[7] Mata, E. G. Phosphorus, Sulfur Silicon Relat. Elem. 1996, 117, 231.

[8] Carreno, M. C. Chem Rev. 1995, 95, 1717. 
[9] Paquette, L. A. Synlett 2001, 1.

[10] Madesclaire, M. Tetrahedron 1988, 44, 6537.

[11] Nicolaou, K. C.; Koumbis, A. E.; Snyder, S. A.; Simonsen, K. B. Angew. Chem., Int. Ed. 2000, 39, 2529.

[12] (a) Herradura, P. S.; Pendola, K. A.; Guy, R. K. Org. Lett. 2000, 2 , 2019.

(b) Firouzabadi, H.; Iranpoor Gholinejad, M. Adv. Synth. Catal. 2010, 352, 119 .

[13] Wendeborn, S.; De Mesmaeker, A.; Brill, W. K.-D.; Berteina, S. Acc. Chem. Res. 2000, 33, 215.

[14] Savarin, C.; Srogl, J.; Liebeskind, L. S. Org. Lett. 2002, 4, 4309.

[15] Hu, B. L.; Hu, H. N.; Sun L. L.; Tang, R. Y. Chin. J. Chem. 2012, $30,2556$.

[16] (a) Kumar, P.; Pandey, P. K.; Hegde, V. R. Synlett 1999, 1921.

(b) Chelucci, G.; Culeddu, N.; Saba, A.; Valenti, R. Tetrahedron: Asymmetry 1999, 10, 3537.

(c) Lou, F.-W.; Zhou, J.-F.; Lin, X.-F.; Chen, Z.-C. Acta Chim. Sinica 2010, 68, 1223 (in Chinese).

(娄凤文, 周建峰, 林贤福, 化学学报, 2010, 68, 1223.)
[17] (a) Falck, J. R.; Lai, J.-Y.; Cho, S.-D.; Yu, J. Tetrahedron Lett. 1999, 40, 2903.

(b) Garofalo, A.; Campiani, G.; Fiorini, I.; Nacci, V. Tetrahedron 1999, 55, 1479.

(c) Shibata, K.; Yamaga, H.; Mitsunobu, O. Heterocycles 1999, 50, 947.

(d) Palomo, C.; Oiarbide, M.; Lopez, R.; Gomez-Bengoa, E. Tetrahedron Lett. 2000, 41, 1283.

(e) Zaragoza, F. Tetrahedron 2001, 57, 5451.

[18] (a) Zaragoza, F.; Stephensen, H. Angew. Chem., Int. Ed. 2000, 39, 554.

(b) Dowsland, J.; McKerlie, F.; Procter, D. J. Tetrahedron Lett. 2000, 41, 4923.

[19] Bell, T. N.; Kirszensztejn, P.; Czajka, B. Catal. Lett. 1995, 30, 305

[20] Hu, Y.-H.; Gao, J.; Wang, J.; Dong, L.; Ding, W.-P.; Chen, Y. Chem. J. Chin. Univ. 2001, 22, 1735 (in Chinese).

(胡玉海, 高健, 王军, 董林, 丁维平, 陈懿, 高等学校化学学报, 2001, 22, 1735.) 